\title{
The role of acupuncture in controlling the gagging reflex using a review of ten
} \section{cases}

\author{
J. Fiske, ' and C. Dickinson, ${ }^{2}$
}

The gagging reflex is a physiological reaction which safeguards the airway from foreign bodies. In some people this response is exaggerated to the extent that the acceptance/provision of dental treatment is not possible. The aim of this paper is to review the role of acupuncture in controlling gagging as a safe, cheap, quick and relatively non-invasive technique.

Method: Ten people agreed to try ear acupuncture to control gagging during dental treatment. Prior to treatment the severity of gagging was assessed. Acupuncture needles were inserted into a specific anti-gagging point on each ear, manipulated briefly and left in situ. Dental treatment was then carried out and the effectiveness of the acupuncture in preventing gagging was assessed. After treatment, the needles were removed and the patient discharged. All acupuncture was carried out by a dentist trained in its use.

Results: Four people had a severe gag reflex which made treatment impossible and six had a very severe reflex which made treatment impossible and affected their dental attendance. Ear acupuncture completely controlled the gag reflex in eight cases (23 treatment episodes) and partially controlled the reflex in two cases (two treatment episodes). Dental treatment could be carried out in all cases and at all visits. The cost of materials was $\mathfrak{E} 0.2$ per person per visit. Additional clinical time was in the order of 2-3 minutes. There were no adverse reactions to the technique and, on all occasions, patients were fit to leave the surgery and travel home unaccompanied.

Conclusions: Ear acupuncture was successful in controlling the gag reflex. It is a safe, quick, inexpensive and relatively noninvasive technique. A controlled clinical trial is required to investigate any placebo effect.

G agging has been defined as an ejectory contraction of the mus$\mathbf{J}_{\text {cles of the pharyngeal sphincter }}{ }^{1}$. It is a normal protective reflex designed to protect the airway and remove irritant material from the posterior oropharynx and the upper gastrointestinal tract. In some individuals this protective reflex is exaggerated causing difficulties in the provision and acceptance of routine dental treatment.

\section{Aetiology of gagging}

The aetiology of gagging has been categorized as somatic or psychogenic ${ }^{2}$. Somatic gagging is induced by touching a trigger area in the oral cavity such as the lateral border of the tongue or the

\footnotetext{
${ }^{1 *}$ Senior Lecturer and Honorary Consultant in Special Care Dentistry, ${ }^{2}$ Clinical Assistant, Department of Sedation and Special Care Dentistry Floor 26, Guy's

Tower, London SE1 9RT

${ }^{*}$ Correspondence to: J Fiske

email:janice.fiske@kcl.ac.uk

REFEREED PAPER

Received 29.09.00; Accepted 21.03.01

(c) British Dental Journal 2001; 190: 611-613
}

palate. Psychogenic gagging is induced without direct contact and the thought of the stimulus, such as a dental intervention, is sufficient to induce the reflex. A single aetiological factor is too simplistic an explanation. For example, somatic gagging induced by a dental mirror placed in the mouth should, in theory, be reproduced by other objects. However, many people who gag at the dentist can eat, brush their teeth and place other objects in their own mouths with little or no consequence.

Other factors have been described which are not necessarily direct inducers of gagging, but which increase its risk and its severity in affected people. These contributing factors include anatomical variations, for example in soft palate anatomy ${ }^{3}$; medical conditions, such as nasal obstruction ${ }^{4}$; psychological factors, such as apprehension $^{5}$ and neuroticism ${ }^{6}$; and dental or iatrogenic factors, such as faults in denture design related to tongue space, retention, position of the post dam and freeway space. ${ }^{7,8}$ Ramsey et al ${ }^{9}$ postulate that the important factors are the past dental experiences and the learned response. That is to say, that people who have had a bad dental experience in the past expect to gag, either consciously or sub-consciously, during future similar events. Consequently their gagging becomes a conditioned response to the dental situation. Ramsay et $a l^{9}$ believe that it is only the severity of the response that distinguishes whether the person actually gags when something is placed in the mouth or at just the thought of it.

\section{Management of gagging}

Some people avoid dental treatment altogether because of their problematic gagging reflex, whilst others have to accept sedation or general anaesthesia in order to cope with dental treatment. A number of strategies have been used in an attempt to control gagging in the dental setting so that patients can cope with treatment and dentists can provide it. They include relaxation, distraction and desensitisation, psychological and behavioural techniques, sedation, and complementary therapies such as hypnosis and acupuncture ${ }^{10}$.

\section{Aim}

The aim of this paper is to illustrate the role of acupuncture as a method of controlling the gag reflex which is safe, quick, inexpensive and relatively non-invasive.

Acupuncture is a system of medicine in which a fine needle is inserted through the skin to a depth of a few millimeters, left in place for a time, sometimes manipulated and then withdrawn. There are two philosophies - that of traditional eastern or Chinese acupuncture which is an integral part of traditional Chinese medicine and that of western scientific approach. According to Filshie and Cummings, the principal difference between these two approaches is "the method of diagnosis and the relentless pursuit of a scientific rationale for treatment". ${ }^{11}$ Central to the scientific approach is the formulation of a diagnosis based on best current practice and the selection of the most appropriate therapeutic option. Acupuncture is one of a range of treatment options that can 


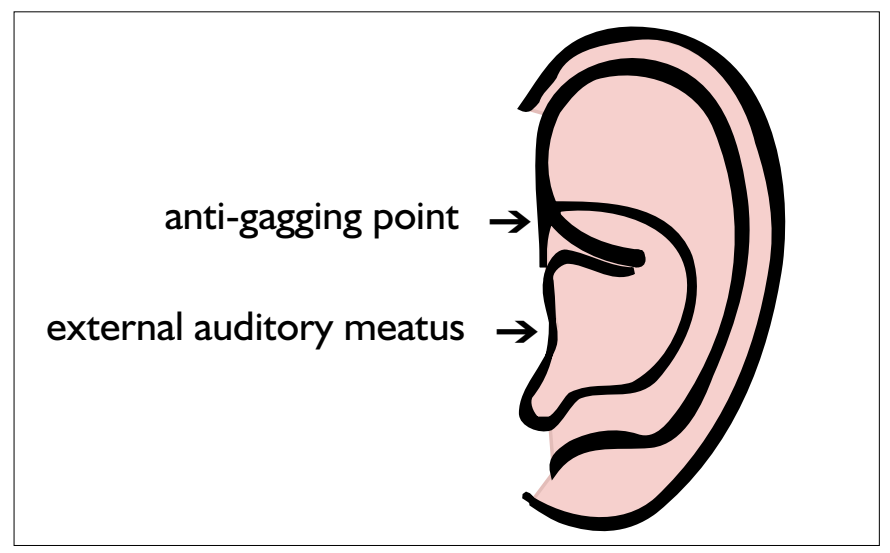

Fig. I Anti-gagging point on ear

be employed in an effort to control gagging.

\section{Method}

Ear acupuncture was selected for the following reasons:

- There is a specific, recognised anti-gagging point on the ear (Fig. 1)

- The needles are not disturbed during access to the mouth for dental treatment

- The needles are out of the patient's line of vision - a bonus for anyone with a dislike of needles

The technique involves the insertion of one, fine, single-use disposable needle of $7 \mathrm{~mm}$ length into the anti-gagging point of each ear to a depth of $3 \mathrm{~mm}$. The needles are manipulated for 30 seconds prior to carrying out dental treatment. The needles remain in situ throughout treatment and are removed before the patient is discharged. The patient does not require an escort and is not inconvenienced in any way following treatment.

Ten people, with an exaggerated gag reflex, who had been referred to a specialist sedation unit because their gag reflex prevented conventional dental treatment agreed to try ear acupuncture to control the reflex. The technique was explained and informed consent obtained in all cases. The magnitude of the gag reflex was assessed using the Gagging Severity Index (GSI) ${ }^{10}$ prior to acupuncture (Table 1). The acupuncture needles were inserted, as described above, and dental treatment commenced. The Gagging Prevention Index $(\mathrm{GPI})^{10}$ was used to assess the effectiveness of the ear acupuncture in controlling the reflex and allowing dental treatment to take place (Table 2). On the completion of treatment, the needles were removed. All acupuncture treatments were carried out by one dentist trained in dental acupuncture.

\section{Results}

Eight men and two women had ear acupuncture in an effort to control their gag reflex so that they could accept dental treatment. Their ages ranged from $40-65$ years.

Using the GSI, four people rated IV with a severe reflex that made treatment impossible and six people rated $\mathrm{V}$ with a very severe reflex which made treatment impossible and affected their dental attendance (Fig. 2). Prior to referral, two people had avoided treatment,

Table 2 Gagging prevention index (GPI)

Treatment management method employed:

I. Obtunded gag reflex; treatment successful

II. Partially controlled gag reflex; all treatment possible

III. Partially controlled gag reflex but frequent gagging; simple treatment possible

IV. Inadequately controlled gag reflex; simple treatment unable to be completed

V. Gag reflex severe; no treatment possible.

Source: Dickinson, 2000
Table I Gagging severity index (GSI)

The gagging reflex is:

I Very mild, occasional and controlled by the patient

II. Mild, and control is required by the patient with reassurance from the dental team

III. Moderate, consistent and limits treatment options

IV. Severe and treatment is impossible

V. Very severe; affecting patient behaviour and dental attendance and making treatment impossible.

Source: Dickinson, 2000

six people were only able to receive treatment with intravenous sedation and two people had not been able to receive treatment at all because of their exaggerated gag reflexes.

Using ear acupuncture, these ten people underwent a total of 25 dental treatment episodes. Procedures carried out included scaling, root planing, impressions, fillings, extractions, adhesive bridge placement, a molar endodontic treatment and a biopsy of the lateral border of the tongue.

The cost of materials for ear acupuncture was $\mathfrak{E} 0.2$ or 0.3 Euros per person per treatment episode. After the initial explanation, the additional time per visit was in the order of $2-3$ minutes. There were no adverse reactions to the technique and, on all occasions, patients were fit to leave the surgery and travel home unaccompanied.

Using the GPI to assess the effectiveness in controlling the groups' gag reflexes, for eight people and 23 episodes of treatment the score was I with the reflex obtunded and dental procedures carried out successfully (Fig. 3). For two people and two episodes of treatment the score was II with the reflex partially controlled and dental treatment still possible.

\section{Discussion}

\section{Gagging}

The aetiology of gagging is complex and not fully understood. Whether its aetiology is somatic, psychogenic or a combination of the two, the outcome is to make the acceptance of dental treatment impossible for some people. Participants in the current review had 'tried everything' to control their gag reflexes in the dental situation. Some had resorted to treatment with intravenous sedation which is an invasive technique using a sedative drug (commonly, Midazolam which has an elimination half-life of two hours ${ }^{12}$ ). Intravenous sedation requires the person to have an escort and affects their ability to carry out certain activities, eg driving, work, etc. The manufacturers of Midazolam recommend that these activities are avoided for eight hours post-administration of the drug. ${ }^{13}$ Other people had avoided dental treatment completely and worried how they would cope if they had a dental emergency. On the occasions when they did try to cope with dental treatment the inability to control their gagging resulted in embarrassment and 'feeling like a child'.

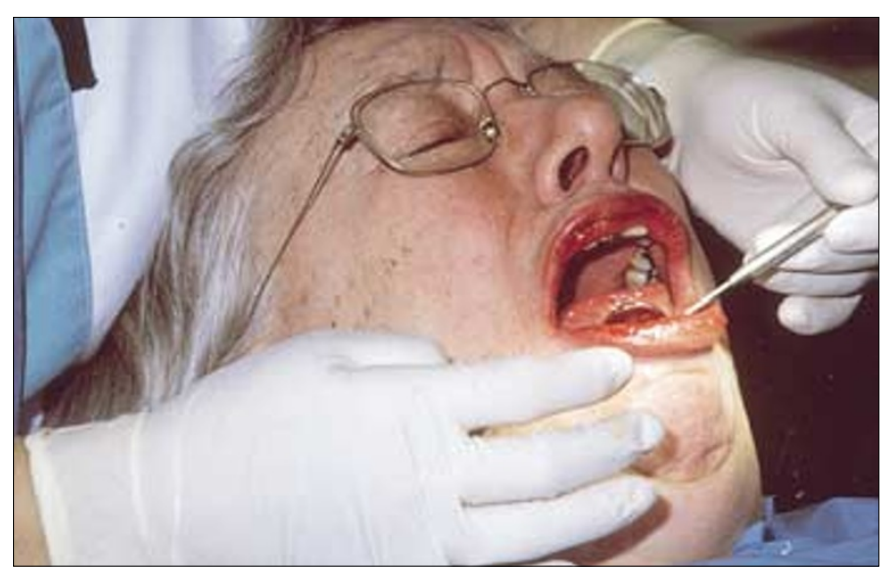

Fig. 2 Patient gagging prior to ear acupuncture 


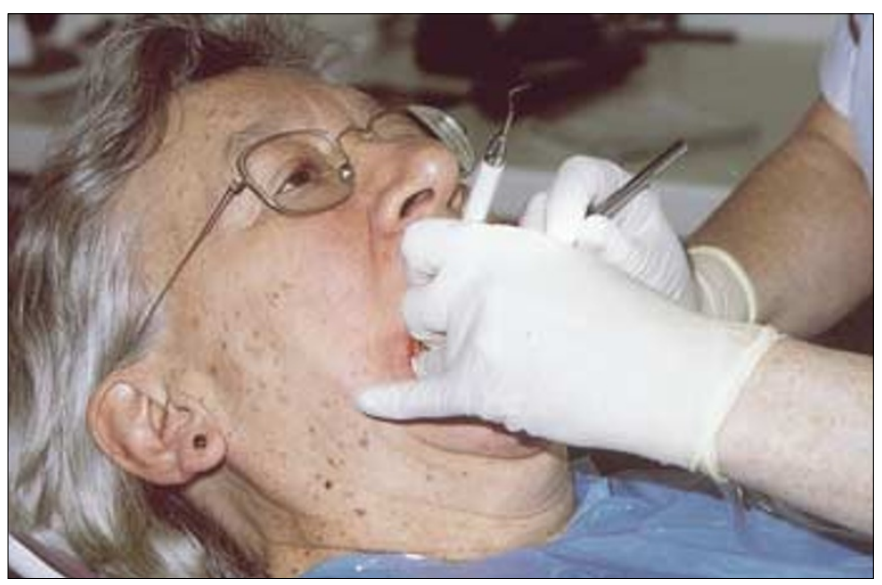

Fig. 3 Patient with acupuncture needles in the ears, receiving dental treatment calmly and comfortably (needle can be seen above patient's earing)

\section{Review of the technique}

Ear acupuncture helped all ten people to accept dental treatment comfortably. However, no account has been taken of any placebo effect. The authors have been unable to find any reference in the dental literature to the previous use of this technique for controlling the gag reflex. A systematic review of the evidence related to the treatment of nausea and vomiting (due to surgery, chemotherapy and pregnancy) concludes that acupuncture is an effective technique in reducing both nausea and vomiting. ${ }^{14}$ In 27 of the 29 trials reviewed, acupuncture was significantly superior to the placebo. ${ }^{14}$ No reference could be found to the use of acupuncture as a longterm preventive treatment for nausea.

The technique of ear acupuncture is relatively non-invasive. It causes little discomfort, is cheap and requires little additional clinical time. The patient is unaffected by the technique, does not require an escort and can return to normal daily activities after treatment. However, it is important that informed consent is gained prior to treatment. The increased public awareness of therapies complementary to medicine meant that all the participants had some prior knowledge of the technique. Although a number of people in this review expressed doubt that the technique would be helpful, they were all agreeable to trying the method. Their readiness to try the technique may have been a reflection of their desperation for a cure.

Rosted ${ }^{15}$ describes acupuncture as a very safe technique provided basic anatomy and aseptic procedures are applied by an appropriately trained practitioner. In this instance the acupuncture was carried out by a dentist with a diploma in dental acupuncture. Information on training can be obtained from the British Dental Acupuncture Society (see Useful Address).

\section{Possible mechanisms of action}

The neurophysiology of acupuncture analgesia is well explained ${ }^{16}$ while that of ear acupuncture in controlling the gag reflex is not. Indeed, the traditional explanation of ear acupuncture is at odds with any scientific basis. It depicts different points on the ear, which represent various body parts equivalent to those of a foetus mapped on the external helix and lobe of the ear. Interestingly, the anti-gagging point on the ear does not correspond to the anatomical representation of the head on the lobe of the ear. However, it does correspond with the area of skin of the external auditory meatus innervated by the auricular branch of the vagus nerve, and is adjacent to an area innervated by the auriculo-temporal branch of the mandibular division of the trigeminal nerve. The vagus nerve is related to swallowing and gagging. Between them, the vagus and the trigeminal nerves are responsible for much of the sensory innervation of the laryngeal, pharyngeal, palatal and oral musosa, and the motor innervation of the musculature of the larynx, pharynx and palate.

In nine of the ten cases in this review, once the acupuncture needles were in place, it was possible to trail a dental mirror across the hard and soft palates. Although the individuals could feel and locate the mirror, no gagging occurred. One can only speculate that in some way, as yet not understood, stimulation of the antigagging points activates mechanisms that inhibit the muscle activity of the gag reflex.

\section{Placebo effect}

The placebo or suggestive effect of treatment can be very powerful. A recent systematic review has confirmed that the placebo effect is mediated via endogenous opioids. ${ }^{11}$ Also, the therapeutic response depends on the complicated interaction of patient factors and expectations, operator factors and expectations, and treatment factors. ${ }^{11}$ The current report is based on clinical cases and not a planned clinical trial, thus no attempt was made to control for the effect of suggestion. The people involved were desperately seeking a solution, and had done so, unsuccessfully, over many years. As one person said when challenged by a friend that she, 'just wanted it to work', 'I have wanted something to work for the last twenty years'. However, there is a need to investigate the placebo effect. A controlled clinical trial is planned in order to do this.

There is also scope to investigate if acupuncture can be helpful as a long-term preventive therapy that would remove the individual's dependency on its use to facilitate dental treatment.

\section{Conclusion}

Ear acupuncture was $100 \%$ successful in controlling the gag reflex in the ten cases reviewed and it was not necessary to compromise dental treatment. The technique is safe, quick, inexpensive and relatively non-invasive. There is a need to investigate the placebo effect.

\section{Useful Address}

British Dental Acupuncture Society (BDAS), Dr Tom Thayer, Trafalgar House, 14 Barker Street, Nantwich, Cheshire CW5 5SY.

1 Savage R D, McGregor A R. Behaviour therapy in prosthodontics. J Prosthet Dent 1970; 24: 126-132.

2 Saunders R M, Cameron J. Psychogenic gagging: identification and treatment recommendations. Compendium of Continuing Education in Dentistry 1997; 18: 430-433.

3 Mack A O. Complete dentures. Br Dent J 1964; 116: 426-429.

4 Wright SM. Medical history, social habits and individual experiences of patients who gag with dentures. J Prosthet Dent 1982; 42: 474-478.

5 Barsby MJ. The control of hyperventilation in the management of gagging. Br Dent J 1997; 182: 109-111.

6 Wright S M. An examination of the personality of dental patients who complain of retching with dentures. Br Dent J 1980; 148: 211-213.

7 Faigenblum MJ. Retching, its causes and management in prosthetic practice. Br Dent J 1986; 125: 485-490.

8 Levine M. Gagging, A problem in prosthondontics. J Can Dent Assoc 1960; 26: 70-75.

9 Ramsay D S, Weinstein P, Milgrom P, Getz M S. Problematic gagging. Principles of treatment. J Am Dent Assoc 1987; 114: 178-183.

10 Dickinson C. Gagging Problems in Dental Patients: Literature Review for the Diploma in Dental Sedation. 2000: GKT Dental Institute of King's College London.

11 Filshie J, Cummings M. Western medical acupuncture . In: Acupuncture a Scientific Appraisal. Ernst E \& White A (Eds). 1999; ButterworthHeinemann: Oxford. 35-59.

12 Association of British Pharmaceutical Industries. Hpnovel. Compendium of Data Sheets and Summaries of Product Characteristics 1998-1999; Datapharm Publications Ltd: London. 1132-1133.

13 Meechan J G, Robb N D, Seymour R A. Pain and Anxiety Control for the Conscious Dental Patient. 1998; Oxford: University Press. 321-322

14 Vickers A J. Can acupuncture have specific effects on health? A systematic review of acupuncture antiemesis trials. JR Soc Med 1996; 89: 303-311.

15 Rosted P. Introduction to acupuncture in dentistry. Br Dent J 2000; 189 136-140.

16 White A. Neurophysiology of acupuncture analgesia. In: Acupuncture a Scientific Appraisal. Ernst E \& White A (Eds). 1999; ButterworthHeinemann: Oxford. 60-92. 